\title{
An Empirical Study on Similarity Functions: Parameter Estimation for the Information Contrast Model
}

\author{
Enrique Amigó and Julio Gonzalo \\ nlp.uned.es \\ ETSI Informática, UNED, \\ Juan del Rosal 16, 28040 Madrid, Spain \\ \{enrique,julio\}@lsi.uned.es
}

February 19, 2022

\begin{abstract}
Computing Textual Similarity implies, at least, two aspects: how to represent items, and how to compare item representations (similarity functions). In this paper, we focus on the second problem, and we discuss the empirical properties of general similarity functions. We focus on the Information Contrast Model (ICM), a parameterized generalization of Pointwise Mutual Information (PMI) which has optimal theoretical properties but has not been thoroughly tested empirically yet. In this paper, we propose an unsupervised parameter estimation criterion for ICM, and we study the empirical behavior of ICM with respect to traditional similarity functions over different representation models (bag of words and word embeddings) and a diverse set of textual similarity problems, including lexical similarity, sentence similarity and short texts similarity. Our empirical results show that (i) the optimal values for the ICM $\beta$ always lie within the range predicted by the theory, $1<\beta<2$, regardless of the task and the representation method chosen; (ii) our proposed estimator $\hat{\beta}$ closely matches the optimal empirical $\beta$ value. In the experiments, our unsupervised method to fix ICM parameters efficiently predicts the optimal values, and ICM outperforms or at least matches the performance of traditional similarity functions.
\end{abstract}

\section{Introduction}

Similarity is a core aspect of many Artificial Intelligence problems, and pervade research on most Information Retrieval (IR), Natural Language Processing (NLP) and Recommendation tasks. Computing Similarity requires, 
at least, deciding how to represent items and how to compare representations. Most research on similarity focuses on the representation problem: what are the best representation models for each task, and how best to extract representative features for each item. This part of the problem is highly task dependent [1] and heavily driven by data.

But this paper focuses on the second problem: finding general, suitable similarity functions able to operate accurately on as many kinds of items and representation models as possible. Some widely used similarity functions are the cosine, the euclidean distance, Jaccard, Lin's similarity, etc. In this paper, we analyse the effectiveness of similarity functions in relation to their formal properties. A formal understanding of the models should improve explainability and predictability of empirical results, two key properties that, in general, typical IR \& NLP models lack. Our work is a contribution to this line of research.

More specifically, we study the Information Contrast Model (ICM) [2], a parameterized generalization of Pointwise Mutual Information (PMI) which is designed to comply with a set of formal properties which the original PMI does not satisfy. Although these properties restrict the range of parameters [2], the authors do not provide any unsupervised method to estimate the optimal ICM parameter. This is a weakness of ICM with respect to other popular similarity functions which do not have parameters. In this paper, we propose and evaluate a theoretically-grounded unsupervised method to estimate the optimal value for the ICM parameter $\beta$ from raw textual data.

In order to assess ICM performance empirically, we perform a wide range of experiments using texts of diverse nature (words, short texts and microblog messages) and two different representation models (word/sentence embeddings and bag-of-words). Our experiments focus on the empirical validation of the following hypotheses: (i) The empirical optimal ICM parameter values fit in the range restricted by the proposed formal properties, (ii) the parameter estimation $\hat{\beta}$ proposed in this paper matches the empirical optimal $\beta$ value and (iii) $\mathrm{ICM}_{0 a t \beta}$ outperforms traditional similarity functions such as cosine, euclidean distance, Lin's similarity or Jaccard, which do not comply with the formal properties specified in [2].

Note that the goal of this work is to study and compare similarity functions under the same conditions, not to outperform the state of the art for each of the individual datasets used in our experiments. This is out of the scope of our research goals, and would imply optimizing multiple taskdependent aspects such as feature definition, extraction, selection, weighting, and optimization with respect to training data. Our experiments are restricted to a completely unsupervised notion of similarity functions, and focus on comparing similarity functions with standard unsupervised or selfsupervised representations. 


\section{Information Contrast Model (ICM) and Param- eter Estimation}

\subsection{ICM: Definition and Constraints}

The Information Contrast Model (ICM) [2] is a generalization of the wellknown PointWise Mutual Information (PMI) that adds three parameters. Being $\mathrm{x}$ and $\mathrm{y}$ two statistical events:

$$
\operatorname{ICM}_{\alpha_{1}, \alpha_{2}, \beta}(x, y)=\log \left(\frac{P(x, y)^{\beta}}{P(x)^{\alpha_{1}} \cdot P(y)^{\alpha_{2}}}\right)
$$

In terms of Shannon-theory-based Information Quantity $(\mathcal{I}(x)=-\log (P(x)))$, ICM can also be expressed as:

$$
\operatorname{ICM}_{\alpha_{1}, \alpha_{2}, \beta}(x, y)=\alpha_{1} \mathcal{I}(x)+\alpha_{2} \mathcal{I}(y)-\beta \mathcal{I}(x, y)
$$

In this paper we restrict the study to the symmetric version of ICM $\left(\alpha_{1}=\right.$ $\alpha_{2}=1$ ), where $\alpha_{1}$ and $\alpha_{2}$ can be ignored. ICM ranges between $-\infty$ and $\infty$, and it is equivalent to PointWise Mutual Information (PMI) when $\beta=1$. It is also equivalent to the product of conditional probabilities when $\beta=2$. ICM also generalizes the Linear Contrast Model proposed by Tversky under certain assumptions (see Section 5.2).

According to the formula above, self-similarity is $\operatorname{ICM}_{\beta}(x, x)=(2-$ $\beta) \mathcal{I}(x)$. In other words, self-similarity grows with the information quantity when $\beta<2$. This behaviour is similar to PMI, which is exactly the amount of information of $\mathrm{x}: \operatorname{PMI}(x, x)=I(x)$. In other words, unlikely events have more self-similarity than likely events.

If $x, y$ are independent, then $I(x, y)=I(x)+I(y)$, and therefore $\operatorname{ICM}_{\beta}(x, y)=$ $(1-\beta)(\mathcal{I}(x)+\mathcal{I}(y))$. This value decreases with the amount of information of $x$ and $y$ when $\beta>1$. In other words, ICM says that two independent events are less similar if their individual information quantities grow. This behavior differs from PMI, which corresponds to $\beta=1$ and gives a uniform similarity value of 0 to any pair of independent events.

ICM is grounded on a set of formal properties proposed in [2] which reflect Information Access requirements and cognitive studies. For a comparison with alternative property sets, please refer to Section 6 . Let us summarize them. Let be $\mathrm{x}$ and $\mathrm{y}$ two events (sets of outcomes) defined over the same probabilistic sample set:

Property 1 (Identity) An event $\mathrm{x}$ is closer to itself than to any other event:

$$
x \neq y \Longrightarrow \operatorname{Sim}(x, x)>\operatorname{Sim}(x, y)
$$


This first constraint is a relaxed version of maximality. Note that the original PMI does not satisfy identity, in particular when $x \supset y$.

In the following, we will say adding information to refer to a "is_a" or subset relationship between probabilistic events $\left(x^{\prime} \subset x\right)$. For instance, "cellular biology" is a subset event of "biology" in the probabilistic sample space.

Property 2 (Identity Specificity) Adding information increases self similarity:

$$
x^{\prime} \subset x \longrightarrow \operatorname{Sim}\left(x^{\prime}, x^{\prime}\right)>\operatorname{Sim}(x, x)
$$

For instance, the expected similarity between two documents about " $b i$ ology" is lower than between documents talking about "Cellular biology". This property is satisfied by PMI but not by other typical similarity functions such as Jaccard, or geometric distances.

Property 3 (Unexpectedness) Adding unexpected information decreases similarity to a greater extent than expected information. Being $x_{1}, x_{2} \subset x$ :

$$
\text { If } P\left(x_{1} \mid x\right)<P\left(x_{2} \mid x\right) \text { then } \operatorname{Sim}\left(x, x_{1}\right)<\operatorname{Sim}\left(x, x_{2}\right)
$$

For instance, if romantic films have been recommended previously, then films that are tagged in a more specific way with an unexpected tag, such as "Romantic terror" are more likely to improve the diversity than films that are tagged as "Romantic love".

Property 4 (Dependency) If we add information to both items, if the conditional probability increases in both directions, then their similarity increases. Being $x^{\prime} \subset x$ and $y^{\prime} \subset y$ :

$$
\begin{aligned}
& \text { If } P\left(x^{\prime} \mid y^{\prime}\right)>P(x \mid y) \text { and } P\left(y^{\prime} \mid x^{\prime}\right)>P(y \mid x) \\
& \text { then } \operatorname{Sim}\left(x^{\prime}, y^{\prime}\right)>\operatorname{Sim}(x, y) \text {. }
\end{aligned}
$$

This property captures the idea that adding new features to each item (adding specificity) that are different can nevertheless increase the similarity between two information pieces. Note that dependency and unexpectedness properties both require considering statistical dependencies between features. Therefore, measures that are based on set intersections and differences (such as Jaccard or Lin's distance) and geometric distances do not satisfy them. In the formal study of [2], ICM is the only one that satisfies the four properties for the range of values $1<\beta<2$. 


\subsection{Probabilistic Framework}

In order to apply and compare similarity functions over document spaces/datasets, we need a formal framework able to accommodate different features and representation approaches. Let $\mathcal{D}$ be a space of documents (a document collection) and $\Gamma$ the universe of available features. In our framework, a representation function is defined as:

Definition 1 A representation function $f_{\Gamma}: \mathcal{D} \longrightarrow \wp(\Gamma)$ is a function from the document collection to the power set of features.

For instance, a possible representation function $f_{\Gamma}$ of texts in $\mathcal{D}$ returns a bag of words $X$, which is a subset of the entire vocabulary $\Gamma$. Two generality issues must be pointed out. First, "bag of features" is a simplification for language representation, because it does not directly incorporate relationships between features such as order and dependencies. However, as in traditional representation models, structure can still be represented with more complex features such as n-grams, linguistic structures, dependency relationships, etc. The bag-of-features directly allows, on the other hand, to include non-textual features (such as metadata).

Second, the features in the previous definition are binary, in the sense that they either appear or not in the representation. However, the model can be extended to accommodate integer or real values via multi-sets or fuzzy sets, respectively. Therefore, the continuous-valued presence of features (as in distributional representations) can also be represented and geometric similarity functions such as euclidean distance or cosine similarity can be captured. Then, similarity functions can be defined as:

Definition 2 A similarity function $\operatorname{Sim}: \wp(\Gamma) \times \wp(\Gamma) \rightarrow \mathbb{R}$ is a function from the feature power set to real numbers.

The notion of feature (word, tag, link, etc.) occurrence is crucial in the context of information access systems. In our framework, it is defined as the set of documents for which $X$ is a subset of its representation. For instance, in a bag of words representation model, the occurrence set of $\left\{w_{1}, w_{2}\right\}$ corresponds with the set of documents which contain both words $w_{1}$ and $w_{2}$ :

Definition 3 The occurrence set $O c c(X)$ of a feature set, $X$, is the set of documents $d$ in the space $\mathcal{D}$ whose representation $f_{\Gamma}(d)$ subsumes the feature set $X$ :

$$
O c c(X)=\left\{d \in \mathcal{D} \mid X \subseteq f_{\Gamma}(d)\right\} .
$$


In order to capture Information Theory based similarity functions such as ICM, Pointwise Mutual Information or Lin's similarity, we define a probabilistic space $(\mathcal{D}, \wp(\mathcal{D}), P)$, where $P$ is a probability function over the sample space of documents $\mathcal{D}$. Therefore, the probability of occurrence of a feature set is ${ }^{1}$ :

$$
P(O c c(X))=P\left(\left\{d \in \mathcal{D} \mid X \subset f_{\Gamma}(d)\right\}\right) .
$$

For instance, the text "cellular biology" can be represented as a set of two features whose occurrence set includes all documents containing both words. In this way:

$$
O c c(\{\text { cellular, biology }\}) \subset O c c(\{\text { biology }\})
$$

and therefore:

$$
P(\operatorname{Occ}(\{\text { cellular, biology }\}))<P(\text { Occ }(\{\text { biology }\}))
$$

For the sake of readability, in the following we will denote the occurrence probability $P(\operatorname{Occ}(X))$ of a feature set $X$ simply as $P(X)$. We will denote the information quantity associated to this event $\mathcal{I}(O c c(X))$ as $\mathcal{I}(X)$.

Our objective is to estimate the similarity $\operatorname{sim}\left(X_{1}, X_{2}\right)$ between two information pieces defined as feature sets which occur in a subset of documents in the collection.

\subsection{Unsupervised $\beta$ estimator}

The formal properties imply a suitable range of $\beta$ values, but its optimal value can vary depending at least on the dataset. Here we propose to estimate $\beta$ as the ratio between the average sum of information quantities and the average joint information quantity of all document pairs:

$$
\hat{\beta}=\frac{A v g_{d, d^{\prime} \in \mathcal{D}}\left(\mathcal{I}\left(f_{\Gamma}(d)\right)+\mathcal{I}\left(f_{\Gamma}\left(d^{\prime}\right)\right)\right)}{A v g_{d, d^{\prime} \in \mathcal{D}}\left(\mathcal{I}\left(f_{\Gamma}(d), f_{\Gamma}\left(d^{\prime}\right)\right)\right)} .
$$

This estimator is designed to satisfy the following requisites:

Property 5 [Boundaries] Assuming that the dependency between features is not negative $(P(X, Y) \geq P(X) \cdot P(Y))$, then $\hat{\beta}$ should be in the range $\hat{\beta} \in[1 . .2]$, as required by the formal properties.

\footnotetext{
${ }^{1}$ Note that the novelty in this probabilistic framework is that it defines a document sample space instead of a feature sample space.
} 
Property 6 [Neutrality] Document pairs that match the expected single and joint information quantities in the collection should receive neutral similarity (zero).

$$
\begin{aligned}
& \text { If } \mathcal{I}\left(f_{\Gamma}(d)\right)=\mathcal{I}\left(f_{\Gamma}\left(d^{\prime}\right)\right)=A v g_{d \in \mathcal{D}}\left(\mathcal{I}\left(f_{\Gamma}(d)\right)\right) \\
& \text { and } \mathcal{I}\left(f_{\Gamma}(d), f_{\Gamma}\left(d^{\prime}\right)\right)=A v g_{d, d^{\prime} \in \mathcal{D}}\left(\mathcal{I}\left(f_{\Gamma}(d), f_{\Gamma}\left(d^{\prime}\right)\right)\right) \\
& \text { then the similarity of } d, d^{\prime} \text { should be zero: } \\
& \text { IC } M_{\hat{\beta}}\left(f_{\Gamma}(d), f_{\Gamma}\left(d^{\prime}\right)\right)=0
\end{aligned}
$$

Property 7 [Expected value] The average $\mathrm{ICM}_{\hat{\beta}}$ over all document pairs in the collection should be zero:

$$
E_{d, d^{\prime} \in \mathcal{D}}\left[I C M_{\hat{\beta}}\left(f_{\Gamma}(d), f_{\Gamma}\left(d^{\prime}\right)\right)\right]=0
$$

In summary, the proposed estimator $\hat{\beta}$ is designed to satisfy the formal properties, give an average similarity value of zero (neutral) and also give neutral similarity to document pairs with average single and joint information quantities.

\subsection{Evaluation Framework}

We have performed a wide range of experiments covering different similarity tasks and representation features, in order to compare ICM performance with traditional similarity functions, and test the predictive power of the proposed $\beta$ estimator. The design and outcome of the experiments is detailed in sections 3, 4 and 5. Our experiments include:

Word co-occurrence: In this experiment, information pieces are words represented as single features. Their probability of occurrence and coocurrence is estimated over the Flickr collection. The task is to predict word similarity as annotated by humans in the WordSim353 corpus [3]. ICM is compared with Pointwise Mutual Information $\left(\mathrm{PMI}=\mathrm{ICM}_{\beta=1}\right)$.

Word embeddings: In this experiment, the information pieces to be compared are words and sentences, represented as distributional semantic vectors (embeddings). The tasks are predicting word similarity values annotated in the corpus MEN [4] and sentence similarities annotated in the STS corpus [5]. We also study a ranking task in which a word definition has to be matched with 15.000 candidate words. In these experiments, the reference similarity functions are PMI and geometric distances (cosine similarity and euclidean distance).

Bag-of-Words: In the third experimental setting, short sentences (tweets) are represented as bags of words. The two tasks consist in identifying tweets 


\begin{tabular}{|c|c|c|}
\hline Word 1 & Word 2 & Similarity \\
\hline baby & mother & 7.85 \\
bread & butter & 6.19 \\
coast & hill & 4.38 \\
cup & object & 3.69 \\
\hline
\end{tabular}

Table 1: Examples of similarity annotation in WordSim353

(i) referring to the same brand and (ii) related to the same topic, in the context of the online reputation monitoring test collection RepLab 2013 [6]. That is, text to text similarity at coarse and fine-grained levels. ICM is compared with PMI and set-based similarity functions such as Jaccard or Lin's distance.

\section{Experiments on Word Co-Ocurrence}

In our first experiment, the task is to estimate lexical similarities for word pairs in WordSim353 [3] using word co-occurrences in documents, i.e. to estimate the similarity $\operatorname{sim}\left(w, w^{\prime}\right)$ between two words $w$ and $w^{\prime}$, given $P(w)$, $P\left(w^{\prime}\right)$, and their joint probability $P\left(w, w^{\prime}\right)$. We use PMI (or $\operatorname{ICM}_{\beta=1}$ ) as reference similarity function. Note that cosine, euclidean distance or Jaccard can not be directly applied in this experiment, given that information pieces (i.e. single words) are not represented as feature sets or vectors.

The dataset contains 353 word pairs, each of which is associated with an average of 13 to 16 human judgements. Annotations (as in all other datasets that are used in this paper) are symmetric by design. Table 1 illustrates some examples of annotated similarities between words in WordSim353. In general, the sampled words are common terms with relatively low ambiguity and somehow agnostic to particular domains. The word pairs also include some specificity relationships (for instance, object and cup).

We compute ICM using direct co-occurrence statistics extracted from the Flickr search facility 2 . For instance, for the words "healthy food", Flickr finds 9,483 documents. Since Flickr stores approximately 13,000 million photos, this represents a probability of $0.729 \times 10^{-6}$. We estimate $\hat{\beta}$ according to the method described in Section 2.3. For this, we calculate the average sum and joint information quantity of word pairs in the data set. The result of the estimation is $\hat{\beta}=1.3$.

Figure 1 shows the Spearman correlation between ICM and the manual similarity annotations in the WordSim-353 dataset as a function of the $\beta$

\footnotetext{
${ }^{2}$ Web search engine statistics are larger, but search engines only give approximations on the number of occurrences, which depend on the length of the query. Flickr, on the other hand, gives exact, more stable numbers.
} 


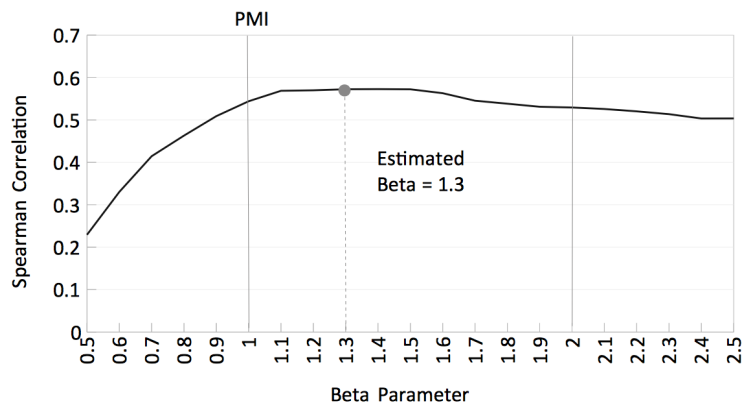

Figure 1: Spearman correlation between ICM (computed with Flickr statistics) and manual similarity assessments in the wordSim353 dataset.

values. We have used a non-parametric correlation coefficient to avoid the effects of scales in similarity estimation and annotation.

Three main observations can be made: First, the optimal empirical $\beta$ values are in the interval $(1.3,1.5)$, within the range predicted by the theory $(1<\beta<2)$. This supports the suitability of the formal properties. Second, the estimator $\hat{\beta}$ matches the empirical optimal $\beta$ values. And, third, all values in the range $[1,1.8]$ outperform PMI (or $\operatorname{ICM}_{\beta=1}$ ). This suggests that satisfying the unexpectedness property (which does not hold for PMI) has empirical effects in this scenario.

\section{Experiments on Word Embeddings}

The most popular - and probably most effective - approach for estimating lexical similarity is to use distributional semantic representations (word embeddings). Here we experiment with the standard skip-bigram with negative sampling and Word2Vec [7]. Word2Vec represents each word $w$ as a vector $\vec{v}_{w}$ that is derived from the co-occurrence statistics of all other words in the corpus (word embeddings). We compare word vectors to estimate their similarity. Word2Vec is closely related to PMI when computing the products of word vectors $[8,9]$.

$$
\left\langle\vec{v}_{w}, \vec{v}_{w^{\prime}}\right\rangle \approx P M I\left(w, w^{\prime}\right)=\log \left(\frac{P\left(w, w^{\prime}\right)}{P(w) \cdot P\left(w^{\prime}\right)}\right) .
$$

Therefore, we can estimate the information quantity of single words as:

$$
\left\langle\vec{v}_{w}, \vec{v}_{w}\right\rangle \approx P M I(w, w)=\log \left(\frac{P(w, w)}{P(w) \cdot P(w)}\right)=\log \left(\frac{1}{P(w)}\right) .
$$

We can also estimate the joint information quantity of two words as: 


$$
\left\langle\vec{v}_{w}, \vec{v}_{w}\right\rangle+\left\langle\vec{v}_{w^{\prime}}, \vec{v}_{w^{\prime}}\right\rangle-\left\langle\vec{v}_{w}, \vec{v}_{w^{\prime}}\right\rangle \approx \log \left(\frac{1}{P\left(w, w^{\prime}\right)}\right) .
$$

According to this, we can estimate $I C M_{\beta}\left(w, w^{\prime}\right)$ in terms of distributional representations as:

$$
\left\langle\vec{v}_{w}, \vec{v}_{w}\right\rangle+\left\langle\vec{v}_{w^{\prime}}, \vec{v}_{w^{\prime}}\right\rangle-\beta\left(\left\langle\vec{v}_{w}, \vec{v}_{w}\right\rangle+\left\langle\vec{v}_{w^{\prime}}, \vec{v}_{w^{\prime}}\right\rangle-\left\langle\vec{v}_{w}, \vec{v}_{w^{\prime}}\right\rangle\right)
$$

In our experiments, we use Word2Vec GoogleNews data [7], which are vectors of 300 dimensions computed over the Google News corpus (3 billion running words).

\subsection{Lexical Similarity}

We now compare ICM in the context of lexical similarity with PMI and with the most widely used similarity criterion with word embeddings: the cosine similarity between word vectors. We compute ICM for the MEN dataset [4] which consists of 3,000 pairs of common words. In this case, similarity is annotated by comparative judgements on two word pairs at a time. We evaluate the results via the same procedure as in the previous experiment. In this case, the estimated $\beta$ according to Equation 2 is $\hat{\beta}=1.16$.

Figure 2 shows the results, which yield higher overall correlations with the manual annotations than in our first experiment. Just like in the previous experiment, the optimal $\beta$ values are located within the values that satisfy the formal properties $(1<\beta<2)$. The estimator $\hat{\beta}$ is very close to the optimal $\beta$ values, and $I C M_{\hat{\beta}}$ outperforms PMI $\left(I C M_{\beta=1}\right)$, the euclidean distance and cosine similarity.

The cosine similarity is a geometric similarity function which does not satisfy identity specificity. Although the improvement of ICM over cosine is slight (0.76 vs 0.73 Spearman correlation), this result may indicate that respecting the property has empirical advantages in this scenario.

\subsection{Sentence Similarity}

We now experiment with a Semantic Textual Similarity task [1] under the Word2Vec representation model. Once each word is represented as a vector, we must decide how to extend the representation to word sequences. Solutions range from simple algebraic operations, such as vector average, to complex deep neural network codifiers that capture Word-order dependencies. As our objective is not to build an optimal system, but rather to compare the behaviour of ICM with other similarity functions, we simply use the vector average, which has been proven to be a strong baseline across multiple tasks [10].

We use the STS dataset [5] which contains 8628 sentence pairs used in the STS tasks organized in the context of SemEval between 2012 and 2017. 


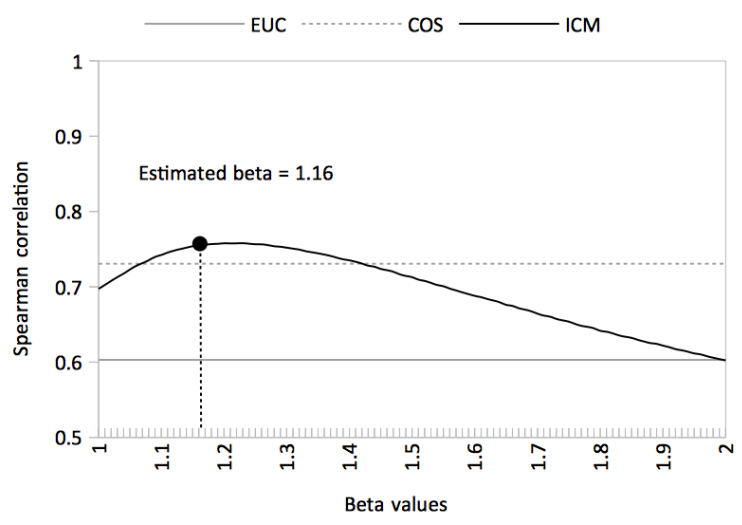

Figure 2: Spearman correlation between ICM computed with word2vec with manual similarity assessments in the MEN dataset.

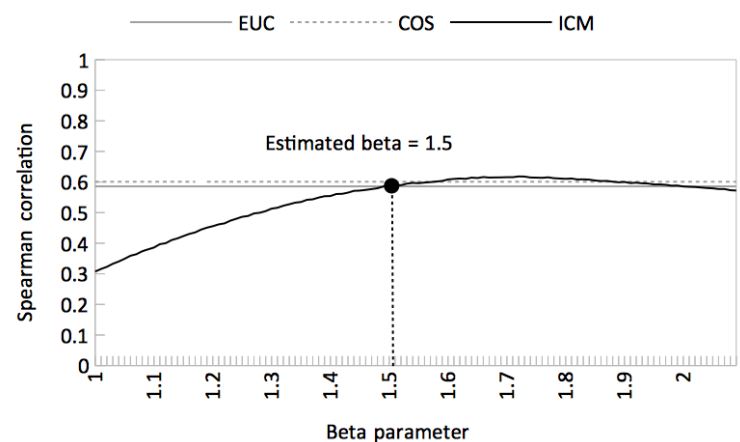

Figure 3: Spearman correlation of ICM between sentences represented as a sum of vectors (word2vec) against manual similarity assessments in STS data set.

This corpus is currently the standard evaluation benchmark for semantic textual similarity. The experiment design is similar to the previous one.

Figure 3 shows the results. Once more, the optimal $\beta$ values are located between 1 and 2 , and the estimated parameter $\hat{\beta}$ is close to the optimal $\beta$ values. In this case, however, $I C M_{\hat{\beta}}$ achieves similar results than the cosine distance and the euclidean distance. Note that in [2] it is proved that cosine similarity under Word2Vec representation satisfies unexpectedness and dependency constraints, but not identity specificity. This result suggests that in this scenario, the specificity of sentences does not have empirical effect.

Indeed, the examples showed in Table 2 illustrate that specificity does not play a significant role in human annotations of similarity. The table shows two similarity instances with maximal annotated similarity: the sen- 


\begin{tabular}{|l|l|}
\hline Text 1 & This is a terrible idea. \\
\hline Text $\mathbf{2}$ & This is a bad idea. \\
\hline \hline Text 1 & $\begin{array}{l}\text { The device plays Internet radio streams and } \\
\text { comes with a 30-day trial of RealNetworks' } \\
\text { Rhapsody music service. }\end{array}$ \\
\hline Text 2 & $\begin{array}{l}\text { The product also streams Internet radio and } \\
\text { comes with a 30-day free trial for RealNetworks' } \\
\text { Rhapsody digital music subscription service. }\end{array}$ \\
\hline
\end{tabular}

Table 2: Two examples with maximal similarity in the STS data set.

\begin{tabular}{c|ccc} 
& ICM & EUC & COS \\
\hline Average Ranking Position & $\mathbf{5 8 0 . 5 7}$ & 6503.92 & 675.41 \\
Average Reciprocal Rank & $\mathbf{0 . 1 7}$ & 0.02 & 0.16 \\
DCG & $\mathbf{0 . 3 1}$ & 0.10 & 0.30 \\
Rank Log & $\mathbf{5 . 0 9}$ & 12.12 & 5.39
\end{tabular}

Table 3: Performance of ICM, euclidean and cosine similarities for the word/definition comparison task.

tences in the first example are less informative (and could provide from very different contexts), and the sentences in the second example are much more informative and specific.

\subsection{Ranking Words by Proximity to Definitions}

In order to test the capability of ICM to reflect the specificity of texts in the similarity estimation, we now define a more objective experiment which does not require human annotations. The task consists in identifying a word from a dictionary given its definition. More specifically, candidate words are ranked according to the similarity between representations.

We consider the words that appear in the MEN corpus and retrieve their first definition in the WordNet ontology [11]. Then, we remove stopwords from the definitions, and represent each definition as the average of their word embeddings. Next, we randomly select 15,000 words from the Word2Vec vector model. We compute the $\hat{\beta}$ estimator for each test case by considering the similarities between the definition representation and the 15,000 candidate word representations. Finally, we sort the 15,000 words plus the word that is being defined according to their similarity to the definition representation. We compare the similarity functions in terms of the rank position of the correct word.

Table 3 shows the comparative effectiveness of ICM, cosine and euclidean 


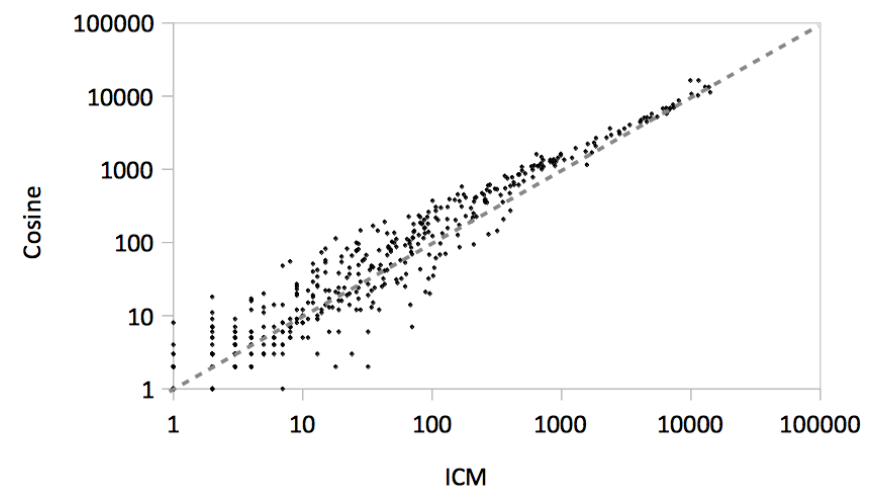

Figure 4: Ranking positions (in logarithmic scale) of the target word given its similarity to the definition: ICM vs. cosine similarity functions.

distance in terms of average ranking position, reciprocal rank $\left(\frac{1}{\operatorname{Rank(w)}}\right)$, DCG $\left(\frac{1}{1+\log (\operatorname{Rank}(w))}\right)$ and rank position in a logarithmic scale $(\log (\operatorname{Rank}(w)))$. ICM outperforms the other two similarity functions in all measures, although the difference with cosine is not statistically significant at the 0.09 level (ttest gives $p=0.09$ for the comparison of ranking positions at log scale).

Figure 4 illustrates the ranking positions of the target word given its similarity to the definition according to ICM and cosine over the average Word2Vec representation. Values are plotted at logarithmic scale. As the figure shows, ICM tends to improve cosine (points over the diagonal).

\section{Experiments on Bag of Words}

We now study the effectiveness of ICM and other similarity functions over Bag of Words (BoW) representations. We first discuss how to compute joint probabilities of word sets (features) under BoW in different ways depending on which statistical assumptions are made; then we present a unified explanation of the most common similarity functions in terms of their statistical assumptions and their relation to Tversky's linear and ratio models; and finally we discuss experimental results on a tweet similarity dataset.

\subsection{Probability Estimations}

The main task when applying ICM and other Information Theory based similarity functions over BoW representations is to compute the Information Quantity $(\mathcal{I}(X)=-\log (P(X))$, with $X$ being the set of terms in a specified text. $\mathcal{I}(X)$ can be estimated in different ways depending on which statistical assumptions are made. 
Independence and Equiprobability: If we assume that terms are equiprobable and statistically independent:

$$
\forall w_{1}, w_{2}\left(P\left(w_{1}\right)=P\left(w_{2}\right) \wedge P\left(w_{1}, w_{2}\right)=P\left(w_{1}\right) \cdot P\left(w_{2}\right)\right)
$$

then the information quantity is proportional to the amount of words. If $c$ is the number of occurrences of any word in the corpus:

$$
\mathcal{I}_{\text {ind }}(X)=-\log \left(\prod_{w_{i} \in X} P\left(w_{i}\right)\right)=\sum_{w_{i} \in X} \log \left(\frac{1}{c}\right) \propto|X|
$$

Word Independence but no Equiprobability: Term specificity (as in idf weighting) is a crucial feature of Natural Language. If we assume that tokens are independent but not equiprobable, the information quantity of a set of tokens can be estimated as:

$$
\begin{aligned}
\mathcal{I}_{\text {ind }}(X) & =-\log (P(X))) \\
& =-\log \left(\prod_{w_{i} \in X} P\left(w_{i}\right)\right)=\sum_{w_{i} \in X} \mathcal{I}\left(w_{i}\right)
\end{aligned}
$$

No Independence and no Equiprobability: Although word independence is sometimes a reasonable simplification, it is sub-optimal and also a poor choice for testing ICM: its strength with respect to other similarity functions is that it incorporates term dependencies - otherwise, it would not satisfy the formal properties. As full joint probabilities cannot be directly computed, we will estimate them using only pairwise word probabilities. For a set of words $X=\left\{w_{1}, . ., w_{n}\right\}$, we use the following estimator:

$$
\mathcal{I}_{\text {dep }}(X)=\frac{\sum_{w_{1}, w_{2} \in X} \mathcal{I}\left(\left\{w_{1}, w_{2}\right\}\right)}{|X|^{\gamma}}
$$

The parameter $\gamma$ is a correction that depends on the characteristics of the dataset. Its boundary values are determined by two extreme possibilities: (i) if words are actually independent, then we should have $\mathcal{I}(X)=\sum \mathcal{I}\left(w_{i}\right)$ and therefore $\gamma$ must be 1; (ii) if words are completely dependent, then we should have $\mathcal{I}(X)=\mathcal{I}\left(w_{i}\right)$, which is satisfied when $\gamma=2$. An estimation of $\gamma$ that complies with these two boundary conditions is the expected ratio of Information Quantities of feature pairs with and without independence:

$$
\gamma=\mathbb{E}_{d \in \mathcal{D}}\left[\frac{\sum_{w_{1}, w_{2} \in f_{\Gamma}(d)} \mathcal{I}\left(\left\{w_{1}\right\}\right)+\mathcal{I}\left(\left\{w_{2}\right\}\right)}{\sum_{w_{1}, w_{2} \in f_{\Gamma}(d)} \mathcal{I}\left(\left\{w_{1}, w_{2}\right\}\right)}\right]
$$




\subsection{Ratio-Based Similarity Functions}

In the context of BoW representation, we want to compare ICM with similarity functions that are based on feature sets (Jaccard) and on Information Theory (Lin's distance and PMI). In this section we explain how popular similarity functions such as Jaccard or Lin's distance are generalized by the Tversky's Ratio Contrast Model under certain statistical assumptions and considering Information Quantity as feature salience function. To cover all possible options, in our experiments we compare ICM with both the ratio and the linear general models proposed by Tversky instantiated with the three statistical assumptions considered in the previous section.

First we consider the Ratio Contrast Model (RCM). Using Information Quantity $(\mathcal{I}(X))$ as salience function and being $X$ and $Y$ two texts represented as word sets, Tversky's RCM is:

$$
\mathrm{RCM}=\frac{\alpha_{1} I(X \cap Y)}{\alpha_{2} I(X \cap Y)+\alpha_{3} I(X \backslash Y)+\alpha_{4} I(Y \backslash X)}
$$

Let us examine the ratio-based similarity measures that result from RCM with different statistical assumptions:

Independence and Equiprobability $\left(\mathbf{R C M}^{i n d}\right.$, Jaccard): When assuming independence and equiprobability $(I(X) \propto|X|)$, most set-based similarity measures can be derived from the ratio contrast model. Ling [12] reported a comprehensive description of these measures. With different values for $\left\langle\alpha_{1}, \alpha_{2}, \alpha_{3}, \alpha_{4}\right\rangle$, we obtain the following measures: Jaccard $(\langle 1,1,1,1\rangle)$, precision $(\langle 1,1,0,1\rangle)$, recall $(\langle 1,0,1,1\rangle)$, the dice coefficient $(\langle 2,1,1,2\rangle)$, the Anderberg coefficient $(\langle 1,2,2,1\rangle)$ and the first Kulczynski coefficient $(\langle 1,1,1,0\rangle)$. We consider the Jaccard configuration which is the most popular similarity function in the context of information access systems $(\vec{\alpha}=\langle 1,1,1,1\rangle)$. Applying the probability estimation described in Equation 3 and the Ratio Contrast Model definition described in Equation 7, we obtain:

$$
\operatorname{RCM}^{\text {ind }}(X, Y)=\frac{|X \cap Y|}{|X \cup Y|}=\operatorname{Jaccard}(X, Y)
$$

Independence but no Equiprobability (RCM ${ }^{i n d}$, Lin): Removing equiprobability, the ratio contrast model generalizes Lin's distance [13] which corresponds to $\left\langle\alpha_{1}, \alpha_{2}, \alpha_{3}, \alpha_{4}\right\rangle=\langle 2,2,1,1\rangle$. Applying the probability estimation with independence assumption (Equation 4 ) and the Ratio Contrast Model, (Equation 7):

$$
\mathrm{RCM}^{i n d}=\operatorname{Lin}(X, Y)=\frac{2 \times \sum_{x \in X \cap Y} I(x)}{\sum_{x \in X} I(x)+\sum_{y \in Y} I(y)}
$$


No Independence and no Equiprobability $\left(\mathbf{R C M}{ }^{d e p}\right)$ :The last variant of the Ratio Contrast Model (RCM) that we consider assumes neither independence nor equiprobability; we use the same parameters as for Jaccard. Applying equations 5 (probability estimation) and 7 (Ratio Contrast Model), we obtain:

$$
\operatorname{RCM}^{d e p}=\frac{\mathcal{I}_{d e p}(X \cap Y)}{\mathcal{I}_{d e p}(X \cap Y)+\mathcal{I}_{d e p}(X \backslash Y)+\mathcal{I}_{d e p}(Y \backslash X)}
$$

\subsection{Linear Based Similarity Functions.}

Assuming symmetricity $(\operatorname{Sim}(X, Y)=\operatorname{Sim}(Y, X))$ and using again Information Quantity as salience function, Tversky's Linear Contrast Model is defined as:

$$
\mathrm{LCM}=\delta \mathcal{I}(X \cap Y)-\mathcal{I}(X \backslash Y)-\mathcal{I}(Y \backslash X)
$$

Unlike the Ratio Contrast Model, even assuming symmetricity LCM is highly sensitive to the $\delta$ parameter, which determines the relative weight of common features versus discriminative features. Just like ICM, the linear model requires a parameter estimation. In fact, LCM and ICM are closely related: LCM is equivalent to ICM if we assume independence between joint and disjoint features. That is, applying set operators we obtain that whenever:

$$
\mathcal{I}(X)=\mathcal{I}(X \backslash Y)+\mathcal{I}(X \cap Y) \wedge \mathcal{I}(Y)=\mathcal{I}(Y \backslash X)+\mathcal{I}(X \cap Y)
$$

then:

$$
\mathrm{LCM}=\mathcal{I}(X)+\mathcal{I}(Y)-\frac{\delta+2}{\delta-1} \mathcal{I}(X \cup Y)=I C M_{\beta=\frac{\delta+2}{\delta-1}}
$$

This connection is relevant for our study, given that we can provide a parameter estimator for Tversky's LCM, and LCM dependency of parameters has made it less popular than ratio-based similarity functions.

Let us now discuss how LCM is computed under different assumptions:

Independence and Equiprobability (ICM ${ }^{i n d}, \mathbf{L C M}^{i n d}$ ): In this case LCM is equivalent to RCM. Applying ICM (Equation 1), the probability estimation with feature independence and equiprobability assumptions (Equation 3) and the Linear Contrast Model (LCM, Equation 9):

$$
\operatorname{ICM}^{\stackrel{i n d}{e q}}=\mathrm{LCM}^{\text {eqd }}=|X|+|Y|-\hat{\beta}_{i n d}^{i q}|X \cup Y|
$$

where, according to equations 2 and $3, \hat{\beta}_{\text {ind }}$ can be estimated as:

$$
\hat{\beta}_{i n d}=\operatorname{Avg}\left(\frac{|X|+|Y|}{|X \cup Y|}\right)
$$


Independence and no Equiprobability $\left(\mathrm{ICM}^{\text {ind }}, \mathbf{L C M}^{\text {ind }}\right)$ : Under these assumptions, LCM and ICM are equivalent. Applying ICM (Equation 1), the probability estimation under feature independence assumption (Equation 4) and LCM (Equation 9):

$$
\begin{aligned}
\mathrm{ICM}^{\text {ind }} & =\mathrm{LCM}^{\text {ind }} \\
& =\sum_{w \in X} \mathcal{I}(w)-\sum_{w \in Y} \mathcal{I}(w)+\hat{\beta}_{\text {ind }} \sum_{w \in X \cup Y} \mathcal{I}(w)
\end{aligned}
$$

where, according to the $\hat{\beta}$ estimator (Equation 2), and probability estimation under feature independence assumption (Equation 4$), \hat{\beta}_{\text {ind }}$ can be estimated as:

$$
\hat{\beta}_{\text {ind }}=\operatorname{Avg}\left(\frac{\sum_{w \in X} \mathcal{I}(w)+\sum_{w \in Y} \mathcal{I}(w)}{\sum_{w \in X \cup Y} \mathcal{I}(w)}\right)
$$

No Independence and no Equiprobability $\left(\mathbf{L C M}^{\text {dep }}\right)$ : Then, using the LCM definition (Equation 9) and the probability estimation described in Equation 5:

$$
\mathrm{LCM}^{\text {dep }}=\mathcal{I}_{\text {dep }}(X)+\mathcal{I}_{\text {dep }}(Y)-\frac{\delta+2}{\delta-1} \mathcal{I}_{\text {dep }}(X \cup Y)
$$

where, applying the $\hat{\beta}$ estimator (Equation 2) and the independence between joint and disjoint features described in Equation 8, the LCM parameter $\frac{\delta+2}{\delta-1}$ can be estimated as:

$$
\begin{aligned}
& \frac{\delta+2}{\delta-1}=\operatorname{Avg}\left(\frac{\mathcal{I}(X)+\mathcal{I}(Y)}{\mathcal{I}(X \cup Y)}\right) \\
& =\operatorname{Avg}\left(\frac{2 \cdot \mathcal{I}_{\text {dep }}(X \cap Y)+\mathcal{I}_{\text {dep }}(X \backslash Y)+\mathcal{I}_{\text {dep }}(Y \backslash X)}{\mathcal{I}_{\text {dep }}(X \cap Y)+\mathcal{I}_{\text {dep }}(X \backslash Y)+\mathcal{I}_{\text {dep }}(Y \backslash X)}\right)
\end{aligned}
$$

In sum, we have six baselines derived from two models, each with three options for statistical assumptions. The first three are based on Tversky's Ratio Contrast Model: $\mathrm{RCM}^{d e p}$, $\mathrm{RCM}^{\text {ind }}$ (which is Lin's similarity) and $\mathrm{RCM}^{i n d, e q}$ (which is the Jaccard coefficient). The other three are based on the Linear Contrast Model: $\mathrm{LCM}^{d e p}, \mathrm{LCM}^{\text {ind }}$ and $\mathrm{LCM}^{\text {ind,eq }}$, each one with its corresponding parameter estimator. ICM is equivalent to LCM ${ }^{\text {ind }}$ and $\mathrm{LCM}^{i n d, e q}$ under the corresponding statistical assumptions.

Note that, in general, ratio-based measures do not satisfy the specificity property. In addition, assuming independence does not satisfy expectedness and dependency properties. $\mathrm{LCM}^{d e p}$ and $\mathrm{RCM}^{d e p}$ fail to satisfy dependency and expectedness due to the independence assumption between common and discriminative feature sets. In the following experiment, we test to what extent such theoretical limitations affect the similarity estimation performance. 


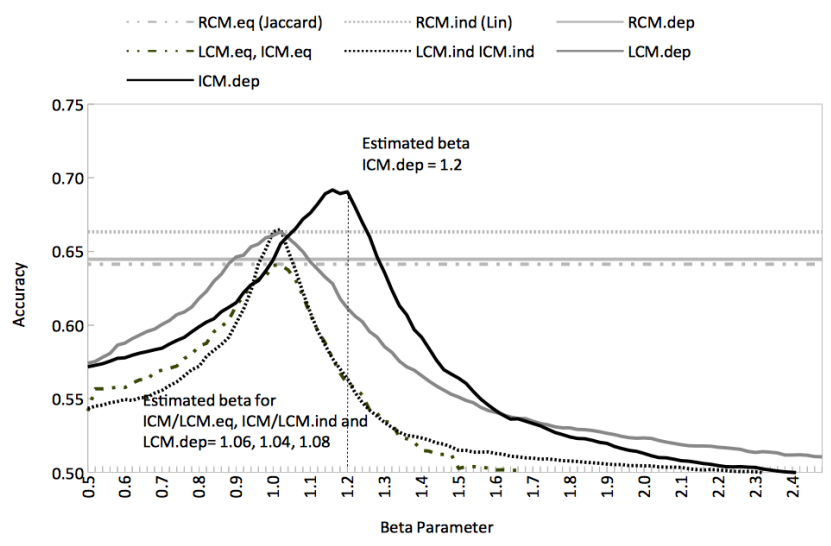

Figure 5: ICM performance for the entity-relatedness task (RepLab 2013 dataset) as a function of various parameter values

\subsection{BoW Experiment: Tweet Similarity in Online Reputa- tion Management.}

For the experiments on BoW, we use the Replab 2013 data set [6]. The dataset consists of a set of tweet streams, each retrieved by using an entity name as a query. It contains data for 61 entities from the automotive, banking, music and university domains. 1,500 tweets per entity were annotated as referring to the entity or not. We use these labels as a coarse-grained binary level of similarity: two tweets about the same entity are more similar than two tweets about different entities. In addition, company-related tweets were also clustered in topics (issues about the entity). We use these clusters as a finer-grained binary notion of similarity: two tweets about the same issue are more similar than two tweets about different issues.

From the annotated tweets, we generated 5,400 similarity test cases. Each test case consists of two tweet pairs $\left(\left(d_{1}, d_{2}\right)\right.$ and $\left.\left(d_{3}, d_{4}\right)\right)$ : in the first pair $\left(d_{1}, d_{2}\right)$, both tweets share the same label; in the second $\left(d_{3}, d_{4}\right)$, they don't. Similarity measures are expected to indicate that the pair of tweets that have the same label are more similar than those that have different labels.

We evaluate the results in terms of accuracy with respect to that decision. Let $f$ denote a similarity function and $t(d)$ the tweet tag that is assigned by humans as the gold standard ("related" or "unrelated"). We compute accuracy as an estimation of:

$$
\operatorname{Acc}(f)=P\left(f\left(d_{1}, d_{2}\right)>f\left(d_{3}, d_{4}\right) \mid t\left(d_{1}\right)=t\left(d_{2}\right) \wedge t\left(d_{3}\right) \neq t\left(d_{4}\right)\right)
$$

In addition to the annotated tweets, the corpus includes a non-annotated set of around 50,000 tweets for each entity; we use this background corpus to 


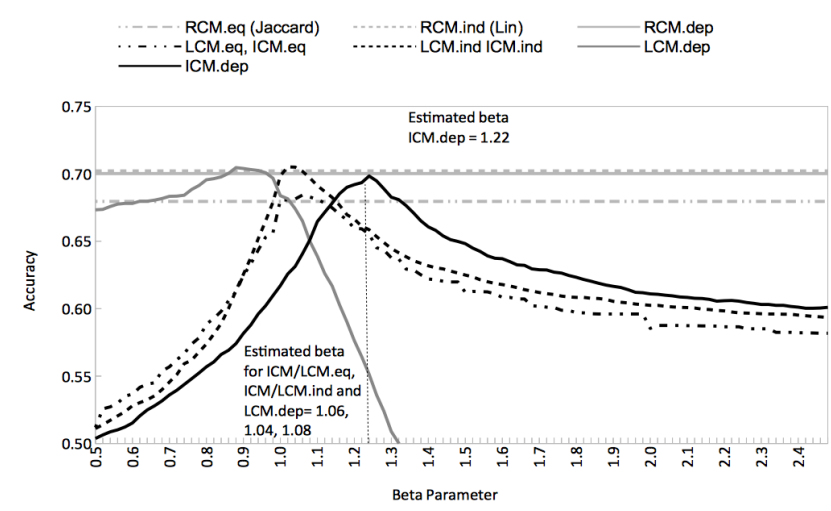

Figure 6: ICM performance for the topic-detection task (RepLab 2013 dataset) as a function of various parameter values

estimate the probability distributions of word co-occurrences for each entity context. The dependency parameter $\gamma$ is estimated according to Equation 6 over tweets in the test data set, obtaining $\gamma=1.25$. The parameter $\beta$ is estimated according to equations $2,13,11$ and 10 for the $\mathrm{ICM}^{d e p}, \mathrm{LCM}$, $\mathrm{ICM}^{i n d}, \mathrm{ICM}^{\text {ind,eq }}$ similarity functions respectively.Figures 5 and 6 show the results of both experiments.

Beta estimation: Just like in previous experiments, $\hat{\beta}$ gets very close to the optimal $\beta$ value for $\mathrm{ICM}^{d e p}$ in both tasks. In addition, the same happens for the estimated $\beta$ under other statistical assumptions (LCM, ICM ${ }^{\text {ind }}$, $\mathrm{ICM}^{i n d, e q}$, which lead to different $\beta$ values, all close to the optimal value in the corresponding function.

ICM performance: In the case of entity-relatedness task, $\mathrm{ICM}^{d e p}$ substantially outperforms all other similarity functions. In the topic detection task, $\mathrm{ICM}^{d e p}$ performance is similar to LCM, RTM ${ }^{d e p}$ and $\mathrm{RTM}^{\text {ind }}$. A possible explanation is the high word overlap in the second task, while the statistical dependencies between intersections and differences is more crucial in the entity-relatedness task. This hypothesis is supported by additional experiments over STS data set and BoW representation, in which sentences are highly overlapped and we obtained similar results.

ICM vs. LCM: In the case of entity-relatedness task, ICM ${ }^{\text {dep }}$ outperforms the Linear Contrast Model. This may imply that the dependency between set intersections and set differences, which is not considered in the Tversky's linear model, has practical implications. 


\section{Related Work}

ICM's formal properties are related to previous axiomatics for similarity. Some of the most popular similarity measures (such as cosine and Euclidean distances) are based on metric space axioms (symmetricity, maximality and triangular inequality). However, both maximality and symmetricity have been already rejected in the context of cognitive science, and are not compatible with the ICM properties [14-16].

Other similarity measures are based on the cognitive transformational model [17], such as WER and PER editing distances [18,19]. Although they do not comply with the unexpectedness and dependency properties, crossobject dependencies can still be captured by considering semantic distances between aligned words in terms of distributional semantics. For instance, some word alignment based approaches have proven to be competitive [20$23]$ in the context of Semantic Textual Similarity tasks. Nonetheless, the alignment processes across objects do not capture the dependencies within each object.

Another approach is to represent objects as probabilistic density functions. Cha et al. [24] describe 65 similarity measures that are based on this framework. The most paradigmatic one is Kullback-Leibler divergence. None of these measures are compatible with unexpectedness and dependency, because inference of statistical dependencies across objects is not possible when objects are modelled as probability distributions.

In 1998, Dekang Lin presented the similarity theorem [13], which is derived from a set of axioms and is closely related to Tversky's ratio model [25]. Its main contribution was the incorporation of the notion of Shannon's Information Quantity, which can be applied in many scenarios. Similar to ICM, Lin's measure (included in our experiments) is derived from informationtheoretic principles and performs competitively in our experiments without the need to adjust any parameter. Cazzanti and Gupta [25], tried to improve Lin's distance by applying the Linear Contrast Model with fixed parameters instead of the Ratio Contrast Model. However, all these models incorporate some form of feature independence assumption between shared and non shared features, which is incompatible with the unexpectedness and dependency properties.

Axioms have also been used in Information Retrieval to develop more accurate retrieval models, as in the seminal work by Fang and Zhai $[26,27]$. Their axioms can be interpreted as a refinement of Tversky's feature model in which the informativeness (discriminative power) of terms is taken into account. As in the case of Tversky's axioms, its main limitation is that they do not consider dependencies between features, and therefore, their axioms are not compatible with the dependency property.

In summary, the main novelty of ICM and the axioms that support it is that they capture feature dependence via the unexpectedness and dependency 
properties. This may lead to better experimental performance when the feature probabilities are indeed dependent, as is the case in many of our experiments.

\section{Conclusions}

ICM is a similarity function which generalizes PMI and is designed to comply with a set of formal properties that (unlike previous similarity axiomatics) model feature dependencies. Just like other linear-based similarity functions, ICM requires a parameter estimation, which is a weakness in comparison with popular ratio-based similarity functions such as Jaccard's coefficient or Lin's similarity. In this paper we have addressed this issue by defining an unsupervised estimator for ICM's $\beta$ parameter, which can also be directly applied to Tversky's Linear Contrast Model.

We have studied the empirical performance of ICM and our $\beta$ estimator, comparing ICM with standard similarity functions based on feature sets (Jaccard), Information Theory (PMI, Lin's similarity), and metric spaces (cosine, euclidean distance). We have compared similarity functions over different representation models (bag of words and word embeddings) and a diverse set of textual similarity problems, including lexical similarity, sentence similarity and short texts similarity.

Three main findings arise from our experiments. The first one is that the results provide an empirical confirmation of the suitable parameter range determined by the ICM formal constraints presented in previous work. In all our experiments, regardless of the representations and the tasks, the optimal $\beta$ range is always located within the boundaries $1<\beta<2$ determined by the formal constraints. These results support the hypothesis that similarity functions can be modeled theoretically according to general principles, abstracting away from the tasks and representation models in which they will be applied.

The second finding is about the predictive power of the unsupervised $\beta$ estimator that we have proposed. In all our experiments, the estimated $\hat{\beta}$ closely matches the optimal empirical $\beta$ value. The additional experiments described in Section 5 also show that $\hat{\beta}$ also approaches optimal $\beta$ values under different statistical assumptions (feature independence and feature equiprobability). This makes the estimator applicable to Tversky's Linear Contrast Model.

The third finding relates to the effectiveness of ICM with the estimated parameter $\hat{\beta}$ with respect to traditional similarity functions. We have found that $\mathrm{ICM}_{\hat{\beta}}$ consistently outperforms the original Point-wise Mutual Information in every experiment. Using word embeddings, we found that the cosine similarity achieves similar results in a test collection where specificity does not play a significant role; but in the lexical similarity benchmark, 
where word specificity plays a role in the similarity annotation, and also when extending this benchmark with word definitions, $\mathrm{ICM}_{\hat{\beta}}$ outperforms cosine.

In the context of bag of words representations, the experimental results suggest that overlap-based similarity functions such as Jaccard or Lin's distance are suitable for comparing information pieces which have high overlap. In other scenarios, such as the coarse text similarity task (entity name disambiguation) $\mathrm{ICM}_{\hat{\beta}}$ provides better results.

In general, our conclusion is that ICM with our $\beta$ estimator either matches or outperforms the effectiveness of similarity functions that do not satisfy the axioms such as PMI, Jaccard distance and Lin's distance, most notably in datasets where feature specificity and dependence affect similarity.

Some additional contributions of our work are (i) a method to estimate ICM between two words using their distributional word representations, which exploits the relationship between word embeddings and PointWise Mutual Information; (ii) a method to compute joint probabilities effectively under a BoW representation that handles feature dependencies; (iii) a typology of similarity functions according to their statistical assumptions and their relation with Tversky's Ratio Contrast Model and Linear Contrast Model.

An open issue is the application of ICM to scenarios in which similarity is asymmetric. Although the original formulation of ICM with three parameters can deal with asymmetricity, the datasets used in our experiments are all symmetric by design. Another open issue is the application of ICM over contextual word embeddings (such as those provided by BERT [28]), which are harder to link with Information Theory notions than Word2Vec non-contextual embeddings.

\section{Appendix: Formal Proofs}

This section contains the formal proofs for the properties of the $\beta$ estimator.

property 5: $[\beta$ Boundaries]. Assuming that the dependency between features is not negative, then:

$$
\begin{aligned}
\forall X, Y(P(X, Y) & \geq P(X) \cdot P(Y)) \\
\Longrightarrow \forall X, Y((I(X, Y)) & \leq(I(X)+I(Y))) \\
\Longrightarrow \frac{\operatorname{Avg}(I(X)+I(Y))}{\operatorname{Avg}(I(X, Y))} & \geq 1
\end{aligned}
$$


In addition,

$$
\begin{aligned}
\forall X, Y(P(X, Y) & \leq P(X)) \\
\Longrightarrow \forall X, Y(I(X, Y) & \geq I(X)) \\
\Longrightarrow \operatorname{Avg}\left(\frac{I(X)+I(Y)}{I(X, Y)}\right) & \leq \operatorname{Avg}\left(\frac{I(X, Y)+I(X, Y)}{I(X, Y)}\right)=2
\end{aligned}
$$

Therefore, $\hat{\beta} \in[1 . .2]$.

Property 6 [Neutrality] Two information pieces with average single and joint information quantity achieve a zero (neutral) similarity. Whenever

$$
\operatorname{Avg}\left(\frac{I(X, Y)+I(X, Y)}{I(X, Y)}\right) \simeq \frac{\operatorname{Avg}(I(X, Y)+I(X, Y)))}{\operatorname{Avg}(I(X, Y)},
$$

if $I(X)=I(Y)=\operatorname{Avg}_{Z \in \Omega}(I(Z))$ and $I(X, Y)=\operatorname{Avg}_{Z, Z^{\prime} \in \Omega^{2}}\left(I\left(Z, Z^{\prime}\right)\right)$ then:

$$
\begin{aligned}
& I C M_{\hat{\beta}}(X, Y)=2 \cdot \operatorname{Avg}_{Z \in \Omega}(I(Z))-\hat{\beta} A v g_{Z, Z^{\prime} \in \Omega^{2}}\left(I\left(Z, Z^{\prime}\right)\right) \\
& \simeq 2 \cdot \operatorname{Avg}_{Z \in \Omega}(I(Z))-2 \cdot \operatorname{Avg}_{Z \in \Omega}(I(Z))=0
\end{aligned}
$$

Property 7 [Expected average] Whenever

$$
\operatorname{Avg}\left(\frac{I(X, Y)+I(X, Y)}{I(X, Y)}\right) \simeq \frac{\operatorname{Avg}(I(X, Y)+I(X, Y)))}{\operatorname{Avg}(I(X, Y)},
$$

then:

$$
\begin{aligned}
& \operatorname{Avg}\left(\operatorname{ICM}_{\hat{\beta}}(X, Y)\right)= \\
& =\operatorname{Avg}(I(X)+I(Y)-\hat{\beta} I(X, Y)) \\
& \simeq \operatorname{Avg}(I(X))+\operatorname{Avg}(I(Y))-\operatorname{Avg}(I(X)+I(Y))=0
\end{aligned}
$$

Therefore, the average $\mathrm{ICM}_{\hat{\beta}}$ across information pieces pairs is zero

\section{Acknowledgment}

\section{References}

[1] E. Agirre, D. Cer, M. Diab, A. Gonzalez-Agirre, and W. Guo, "*sem 2013 shared task: Semantic textual similarity," in Second Joint Conference on Lexical and Computational Semantics ( ${ }^{*}$ SEM), Volume 1: Proceedings of the Main Conference and the Shared Task: Semantic Textual Similarity. Atlanta, Georgia, USA: Association for Computational Linguistics, June 2013, pp. 32-43.

[2] E. Amigó, F. Giner, J. Gonzalo, and F. Verdejo, "On the foundations of similarity in information access," Information Retrieval Journal, vol. 23, no. 3, pp. 216-254, 2020. 
[3] L. Finkelstein, E. Gabrilovich, Y. Matias, E. Rivlin, Z. Solan, G. Wolfman, and E. Ruppin, "Placing search in context: The concept revisited," ACM Transactions on Information Systems, vol. 20, no. 1, pp. 116-131, 2002.

[4] E. Bruni, N. K. Tran, and M. Baroni, "Multimodal distributional semantics," J. Artif. Int. Res., vol. 49, no. 1, pp. 1-47, Jan. 2014. [Online]. Available: http://dl.acm.org/citation.cfm?id=2655713.2655714

[5] D. Cer, M. Diab, E. Agirre, I. Lopez-Gazpio, and L. Specia, "SemEval2017 task 1: Semantic textual similarity multilingual and crosslingual focused evaluation," in Proceedings of the 11th International Workshop on Semantic Evaluation (SemEval-2017). Vancouver, Canada: Association for Computational Linguistics, Aug. 2017, pp. 1-14.

[6] E. Amigó, J. C. de Albornoz, I. Chugur, A. Corujo, J. Gonzalo, T. Martín-Wanton, E. Meij, M. de Rijke, and D. Spina, "Overview of replab 2013: Evaluating online reputation monitoring systems," in Proceedings of Information Access Evaluation. Multilinguality, Multimodality, and Visualization - 4th International Conference of the CLEF Initiative (CLEF 2013), 2013.

[7] T. Mikolov, K. Chen, G. Corrado, and J. Dean, "Googlenews-vectorsnegative300.bin.gz - efficient estimation of word representations in vector space," arXiv preprint arXiv:1301.3781, 2013. [Online]. Available: https://code.google.com/archive/p/word2vec/

[8] O. Levy and Y. Goldberg, "Neural word embedding as implicit matrix factorization," in Proceedings of the 27th International Conference on Neural Information Processing Systems, ser. NIPS'14. Cambridge, MA, USA: MIT Press, 2014, pp. 2177-2185. [Online]. Available: http://dl.acm.org/citation.cfm?id=2969033.2969070

[9] S. Arora, Y. Li, Y. Liang, T. Ma, and A. Risteski, "A latent variable model approach to pmi-based word embeddings," TACL, vol. 4, pp. 385-399, 2016.

[10] T. Kenter, A. Borisov, and M. de Rijke, "Siamese CBOW: Optimizing word embeddings for sentence representations," in Proceedings of the 54th Annual Meeting of the Association for Computational Linguistics (Volume 1: Long Papers). Berlin, Germany: Association for Computational Linguistics, Aug. 2016, pp. 941-951.

[11] C. Fellbaum, WordNet: An Electronic Lexical Database. MIT Press, 1998.

[12] M. H. Ling, "Distance coefficients between two lists or sets," 2010. 
[13] D. Lin, "An information-theoretic definition of similarity," in Proceedings of the Fifteenth International Conference on Machine Learning, ser. ICML '98. San Francisco, CA, USA: Morgan Kaufmann Publishers Inc., 1998, pp. 296-304.

[14] C. Krumhansl, "Concerning the applicability of geometric models to similarity data: The interrelationship between similarity and spatial density." Psychological Review, vol. 85, no. 5, pp. 445-463, 1978.

[15] E. G. Ashby and N. A. Perrin, "Toward a unified theory of similarity and recognition." Psychological review, vol. 95, no. 1, pp. 124-150, 1988.

[16] A. Tversky and I. Gati, "Similarity, separability, and the triangle inequality." Psychological review, vol. 89, no. 2, p. 123, 1982.

[17] U. Hahn, N. Chater, and L. B. Richardson, "Similarity as transformation," Cognition, vol. 87, no. 1, pp. 1-32, Feb. 2003.

[18] S. Nießen, F. J. Och, G. Leusch, and H. Ney, "An Evaluation Tool for Machine Translation: Fast Evaluation for MT Research," in Proceedings of the 2nd International Conference on Language Resources and Evaluation (LREC), 2000.

[19] C. Tillmann, S. Vogel, H. Ney, A. Zubiaga, and H. Sawaf, "Accelerated DP based Search for Statistical Translation," in Proceedings of European Conference on Speech Communication and Technology, 1997.

[20] R. Mihalcea, C. Corley, and C. Strapparava, "Corpus-based and knowledge-based measures of text semantic similarity," in Proceedings of the American Association for Artificial Intelligence (AAAI 2006), Boston, Massachusetts, July 2006.

[21] R. Malik, L. V. Subramaniam, and S. Kaushik, "Automatically selecting answer templates to respond to customer emails." in IJCAI, M. M. Veloso, Ed., 2007, pp. 1659-1664.

[22] L. Han, A. L. Kashyap, T. Finin, J. Mayfield, and J. Weese, "UMBC EBIQUITY-CORE: Semantic Textual Similarity Systems," in Proceedings of the Second Joint Conference on Lexical and Computational Semantics. Association for Computational Linguistics, June 2013.

[23] M. Kusner, Y. Sun, N. Kolkin, and K. Q. Weinberger, "From word embeddings to document distances," in Proceedings of the 32nd International Conference on Machine Learning (ICML15), D. Blei and F. Bach, Eds. JMLR Workshop and Conference Proceedings, 2015, pp. 957-966. [Online]. Available: http://jmlr.org/proceedings/papers/v37/kusnerb15.pdf 
[24] S.-H. Cha, "Comprehensive survey on distance/similarity measures between probability density functions," 2007.

[25] L. Cazzanti and M. Gupta, "Information-theoretic and Set-theoretic Similarity," in 2006 IEEE International Symposium on Information Theory. IEEE, Jul. 2006, pp. 1836-1840.

[26] H. Fang and C. Zhai, "An exploration of axiomatic approaches to information retrieval," in Proceedings of the 28th Annual International ACM SIGIR Conference on Research and Development in Information Retrieval, ser. SIGIR '05. New York, NY, USA: ACM, 2005, pp. 480487.

[27] H. Fang, T. Tao, and C. Zhai, "Diagnostic evaluation of information retrieval models," ACM Trans. Inf. Syst., vol. 29, no. 2, pp. 7:1-7:42, Apr. 2011.

[28] J. Devlin, M.-W. Chang, K. Lee, and K. Toutanova, "BERT: Pretraining of deep bidirectional transformers for language understanding," in Proceedings of the 2019 Conference of the North American Chapter of the Association for Computational Linguistics: Human Language Technologies, Volume 1 (Long and Short Papers). Minneapolis, Minnesota: Association for Computational Linguistics, Jun. 2019, pp. 4171-4186. [Online]. Available: https://www.aclweb.org/anthology/N19-1423 


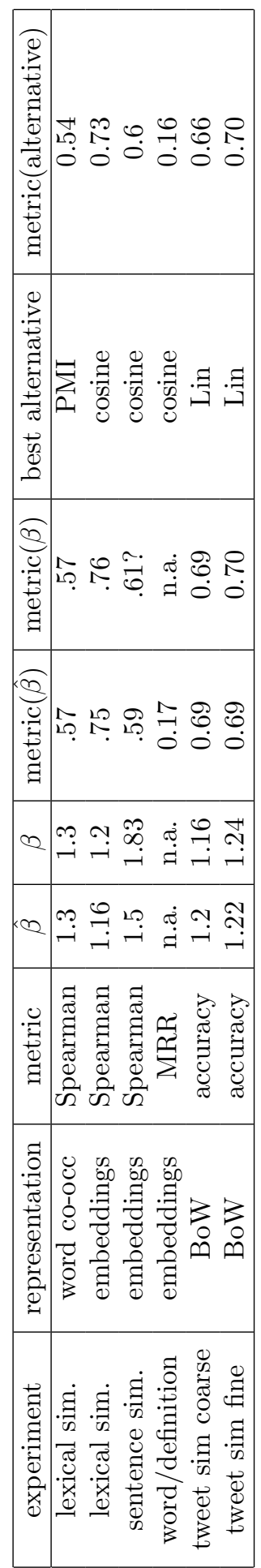

Table 4: Main experimental results. 